\title{
EXISTENCE AND UNIQUENESS OF THE SOLUTIONS OF SOME CLASSES OF INTEGRAL EQUATIONS $C^{*}$-ALGEBRA-VALUED $b$-METRIC SPACES
}

Esad Jakupovića , Hashem P. Masiha ${ }^{b}$, Zoran D. Mitrovićc Seyede S. Razavi ${ }^{\mathrm{d}}$, Reza Saadati ${ }^{\mathrm{e}}$

${ }^{a}$ Academy of Sciences and Arts of the Republic of Srpska, Banja Luka, Republic of Srpska, Bosnia and Herzegovina, e-mail: esadjakupovic50@gmail.com, ORCID ID: (Dhttps://orcid.org/0000-0003-2354-5532

${ }^{\mathrm{b}}$ K. N. Toosi University of Technology, Faculty of Mathematics, Tehran, Islamic Republic of Iran, e-mail: masihahp@kntu.ac.ir, ORCID ID: (1) https://orcid.org/0000-0001-9751-6828

'University of Banja Luka, Faculty of Electrical Engineering, Banja Luka, Republic of Srpska, Bosnia and Herzegovina, e-mail: zoran.mitrovic@etf.unibl.org, corresponding author, ORCID ID: (1) https://orcid.org/0000-0001-9993-9082

${ }^{\mathrm{d}} \mathrm{K}$. N. Toosi University of Technology, Faculty of Mathematics, Tehran, Islamic Republic of Iran, e-mail: ssrazavi@kntu.ac.ir, ORCID ID: (1)https://orcid.org/0000-0002-9772-1140

eIran University of Science and Technology, School of Mathematics, Narmak, Tehran, Islamic Republic of Iran, e-mail: rsaadati@iust.ac.ir, ORCID ID: (1) https://orcid.org/0000-0002-6770-6951

DOI: 10.5937/vojtehg68-28632; https://doi.org/10.5937/vojtehg68-28632

FIELD: Mathematics

ARTICLE TYPE: Original scientific paper

Abstract:

Introduction/purpose: The aim of the paper is to establish some coupled fixed point results in $C^{*}$-algebra-valued b-metric spaces. Moreover, the obtained results are used to define the sufficient conditions for the existence of the solutions of some classes of integral equations.

Methods: The method of coupled fixed points gives the sufficient conditions for the existence of the solution of some classes of integral equations.

Results: New results were obtained on coupled fixed points in $C^{*}$-algebra-valued b-metric space. 
Conclusion: The obtained results represent a contribution in the fixed point theory and open new possibilities of application in the theory of differential and integral equations.

Key words: Coupled fixed point, $C^{*}$-algebra, integral equation.

\section{Basic definitions}

In this section, we review some facts of the $C^{*}$-algebras which are needed in this paper. The references (Ali Abou Bakr, 2019), (Bai, 2016), (Bonsal, 1962), (Hussain et al, 2018), (Huang et al, 2018), (Hussain\&Mitrović, 2017), (Kadelburg et al, 2016), (Kadelburg\&Radenović, 2016), (Kongban\&Kumam, 2018), (Ma et al, 2014), (Ma\&Jiang, 2015), (Mitrović et al, 2019), (Radenović et al, 2017), (Radenović et al, 2019), (Vujaković et al, 2019), (Zoto et al, 2019), (Wu\&Zhao, 2018), (Cao\&Xin, 2016) and (Todorčević, 2019) are useful.

We denote $\mathcal{A}$ as a unital $\mathrm{C}^{*}$-algebra with the unit $1_{\mathcal{A}}$.

Let

$$
\mathcal{A}_{h}=\left\{t \in \mathcal{A}: t=t^{*}\right\}
$$

We say $t \in \mathcal{A}$ a positive element, showed it by $t \succeq 0_{\mathcal{A}}$ if $t \in \mathcal{A}_{h}$ and $\sigma(t) \subseteq[0, \infty)$, where $0_{\mathcal{A}}$ is the zero element in $\mathcal{A}$ and $\sigma(t)$ is the spectrum of $t$.

On the set $\mathcal{A}_{h}$ we have a partial ordering given by $v \succeq u$ if and only if $v-u \succeq 0_{\mathcal{A}}$. Also, we will denote

$$
\mathcal{A}_{+}=\left\{t \in \mathcal{A}: t \succeq 0_{\mathcal{A}}\right\}
$$

and

$$
\mathcal{A}^{\prime}=\{t \in \mathcal{A}: t k=k t \text { for all } k \in \mathcal{A}\} .
$$

Definition 1. Assume that $\mathcal{X} \neq \emptyset$. As usual suppose that $\delta: \mathcal{X} \times \mathcal{X} \rightarrow \mathcal{A}$ is a function fulfilling:

(1) $\delta(u, v) \succeq 0_{\mathcal{A}}$ for each $u$ and $v$ in $\mathcal{X}$;

(2) $\delta(v, u)=0_{\mathcal{A}}$ if $v=u$;

(3) $\delta(u, v)=\delta(v, u)$ for each $u$ and $v$ in $\mathcal{X}$;

(4) $\delta(u, v) \preceq \delta(u, t)+\delta(t, v)$ for each $u$, $v$ and $t \in \mathcal{X}$.

Then $\delta$ is a $C^{*}$-algebra-valued metric (shortly, $C^{*}-A V-M$ ). 
Definition 2. Assume that $\mathcal{X} \neq \emptyset$. Suppose that $b \in \mathcal{A}^{\prime}$ such that $\|b\| \geq 1$. A function $\delta_{b}: \mathcal{X}^{2} \rightarrow \mathcal{A}$ is said to be a $C^{*}$-algebra-valued b-metric (in short $\left.C^{*}-A V-B M\right)$ on $\mathcal{X}$ if for every $u, v, t \in \mathcal{A}$ :

(1) $\delta_{b}(u, v) \succeq 0_{\mathcal{A}}$ for each $u$ and $v$ in $\mathcal{X}$ and $\delta_{b}(u, v)=0$ if $u=v$;

(2) $\delta_{b}(u, v)=\delta_{b}(v, u)$;

(3) $\delta_{b}(u, v) \preceq b\left[\delta_{b}(u, t)+\delta_{b}(t, v)\right]$.

Then $\left(\mathcal{X}, \mathcal{A}, \delta_{b}\right)$ is a $C^{*}-A V-B M$ space with the coefficient $b$.

Example 1 (Ma\&Jiang (2015)). Assume that $\mathcal{X}=\mathbb{R}$ and $\mathcal{A}=M_{n}(\mathbb{R})$. Now, we define

$$
\delta(u, v)=\operatorname{diag}\left(c_{1}|u-v|^{p}, c_{2}|u-v|^{p}, \ldots, c_{n}|u-v|^{p}\right),
$$

where diag denotes a diagonal matrix, and where $u, v \in \mathbb{R}, c_{1}, \ldots, c_{n}$ positive constants and $p \in(1,+\infty)$. It can be shown that $(\mathcal{X}, \mathcal{A}, \delta)$ is a complete $\mathrm{C}^{*}$-AV-BM. We only prove the third statement of Definition 2 . For this we have:

$$
|u-v|^{p} \leq 2^{p}\left(|u-t|^{p}+|t-v|^{p}\right)
$$

then $\delta(u, v) \preceq A[\delta(u, t)+\delta(t, v)]$ for every $u, v, t \in \mathcal{X}$, where $A=2^{p} I \in \mathcal{A}^{\prime}$ and $A>I$ by $2^{p}>1$. Since $|u-v|^{p} \leq|u-t|^{p}+|t-v|^{p}$ is impossible for all $u \succ t \succ v,\left(\mathcal{X}, M_{n}(\mathbb{R}), \delta\right)$ is not a $\mathrm{C}^{*}$-AV-M space.

Definition 3. Assume that $\left(\mathcal{X}, \mathcal{A}, \delta_{b}\right)$ is a $C^{*}-A V-B M$ space. $(u, v) \in \mathcal{X} \times \mathcal{X}$ is called a coupled fixed point (shortly FP) of the function $\psi: \mathcal{X} \times \mathcal{X} \rightarrow \mathcal{X}$ if $\psi(u, v)=u$ and $\psi(v, u)=v$.

For some useful applications, see (Kongban\&Kumam, 2018) and (Ali Abou Bakr, 2019).

\section{Main results}

The following theorem is one of our main results.

Theorem 1. Assume that $\left(\mathcal{X}, \mathcal{A}, \delta_{b}\right)$ is a $C^{*}-A V$-BM space and suppose that $\psi: \mathcal{X}^{2} \rightarrow \mathcal{X}$ is a function satisfying

$$
\delta_{b}(\psi(u, v), \psi(t, s)) \preceq a^{*} \delta_{b}(u, t) a+a^{*} \delta_{b}(v, s) a,
$$

for every $u, v, t, s \in \mathcal{X}$, in which $a \in \mathcal{A}$ with $\|a\|<\frac{1}{\sqrt{2}}$. Then $\psi$ has a unique coupled FP. Moreover, $\psi$ has a unique FP in $\mathcal{X}$. 
Proof. Let $u_{0}, v_{0} \in \mathcal{X}$. Set $u_{1}=\psi\left(u_{0}, v_{0}\right)$ and $v_{1}=\psi\left(v_{0}, u_{0}\right)$. We obtain two sequences $\left\{u_{n}\right\},\left\{v_{n}\right\}$ in $\mathcal{X}$ such that $u_{n+1}=\psi\left(u_{n}, v_{n}\right)$ and $v_{n+1}=\psi\left(v_{n}, u_{n}\right)$ if we continue the above process. From (1) we have

$$
\begin{aligned}
\delta_{b}\left(u_{n}, u_{n+1}\right) & =\delta_{b}\left(\psi\left(u_{n-1}, v_{n-1}\right), \psi\left(u_{n}, v_{n}\right)\right) \\
& \preceq a^{*} \delta_{b}\left(u_{n-1}, u_{n}\right) a+a^{*} \delta_{b}\left(v_{n-1}, v_{n}\right) a \\
& \preceq a^{*}\left(\delta_{b}\left(u_{n-1}, u_{n}\right)+\delta_{b}\left(v_{n-1}, v_{n}\right)\right) a .
\end{aligned}
$$

Similarly,

$$
\begin{aligned}
\delta_{b}\left(v_{n}, v_{n+1}\right) & =\delta_{b}\left(\psi\left(v_{n-1}, u_{n-1}\right), \psi\left(v_{n}, u_{n}\right)\right) \\
& \preceq a^{*} \delta_{b}\left(v_{n-1}, v_{n}\right) a+a^{*} \delta_{b}\left(u_{n-1}, u_{n}\right) a \\
& \preceq a^{*}\left(\delta_{b}\left(v_{n-1}, v_{n}\right)+\delta_{b}\left(u_{n-1}, u_{n}\right)\right) a .
\end{aligned}
$$

Let

$$
\delta_{n}=\delta_{b}\left(u_{n}, u_{n+1}\right)+\delta_{b}\left(v_{n}, v_{n+1}\right) .
$$

From (2) and (3), we have

$$
\begin{aligned}
\delta_{n} & =\delta_{b}\left(u_{n}, u_{n+1}\right)+\delta_{b}\left(v_{n}, v_{n+1}\right) \\
& \preceq a^{*}\left(\delta_{b}\left(u_{n-1}, u_{n}\right)+\delta_{b}\left(v_{n-1}, v_{n}\right)\right) a+a^{*}\left(\delta_{b}\left(v_{n-1}, v_{n}\right)+\delta_{b}\left(u_{n-1}, u_{n}\right)\right) a \\
& =2\left(a^{*}\left(\delta_{b}\left(u_{n-1}, u_{n}\right)+\delta_{b}\left(v_{n-1}, v_{n}\right)\right) a\right) \\
& \preceq(\sqrt{2} a)^{*}\left(\delta_{b}\left(u_{n-1}, u_{n}\right)+\delta_{b}\left(v_{n-1}, v_{n}\right)\right)(\sqrt{2} a) \\
& \preceq(\sqrt{2} a)^{*} \delta_{n-1}(\sqrt{2} a) .
\end{aligned}
$$

Due to the following property: (if $t, k \in \mathcal{A}_{h}$, then $t \preceq k$ implies $u^{*} t u \preceq$ $u^{*} k u$ ), we can obtain for any $n \in \mathbb{N}$,

$$
0_{\mathcal{A}} \preceq \delta_{n} \preceq(\sqrt{2} a)^{*} \delta_{n-1}(\sqrt{2} a) \preceq \ldots \preceq\left((\sqrt{2} a)^{*}\right)^{n} \delta_{0}(\sqrt{2} a)^{n} .
$$

If $\delta_{0}=0_{\mathcal{A}}$, then from (2) of Definition 2 we know $\left(u_{0}, v_{0}\right)$ is a coupled FP of $F$.

Now, by letting $\delta_{0} \preceq 0_{\mathcal{A}}$, we can obtain for $m, n \in \mathbb{N}, n>m$

$$
\begin{aligned}
\delta_{b}\left(u_{n}, u_{m}\right) & \preceq b\left(\delta_{b}\left(u_{n}, u_{n-1}\right)+\delta_{b}\left(u_{n-1}, u_{m}\right)\right) \\
& \preceq b \delta_{b}\left(u_{n}, u_{n-1}\right)+b^{2} \delta_{b}\left(u_{n-1}, u_{n-2}\right)+b^{2} \delta_{b}\left(u_{n-2}, u_{m}\right) \\
& \preceq b \delta_{b}\left(u_{n}, u_{n-1}\right)+b^{2} \delta_{b}\left(u_{n-1}, u_{n-2}\right)
\end{aligned}
$$




$$
\begin{aligned}
& +b^{3} \delta_{b}\left(u_{n-2}, u_{n-3}\right)+\ldots+b^{n-m} \delta_{b}\left(u_{m+1}, u_{m}\right) \\
& \preceq b \delta_{b}\left(u_{n}, u_{n-1}\right)+b^{2} \delta_{b}\left(u_{n-1}, u_{n-2}\right)+\ldots \\
& +b^{n-m-1} \delta_{b}\left(u_{m+2}, u_{m+1}\right)+b^{n-m-1} \delta_{b}\left(u_{m+1}, u_{m}\right) .
\end{aligned}
$$

\section{Similarly,}

$$
\begin{aligned}
\delta_{b}\left(v_{n}, v_{m}\right) \preceq & b \delta_{b}\left(v_{n}, v_{n-1}\right)+b^{2} \delta_{b}\left(v_{n-1}, v_{n-2}\right)+\ldots+ \\
& b^{n-m-1} \delta_{b}\left(v_{m+2}, v_{m+1}\right)+b^{n-m-1} \delta_{b}\left(v_{m+1}, v_{m}\right) .
\end{aligned}
$$

\section{Therefore,}

$$
\begin{aligned}
\delta_{b}\left(u_{n}, u_{m}\right)+\delta_{b}\left(v_{n}, v_{m}\right) & \preceq b\left(\delta_{b}\left(u_{n}, u_{n-1}\right)+\delta_{b}\left(v_{n}, v_{n-1}\right)\right) \\
& +b^{2}\left(\delta_{b}\left(u_{n-1}, u_{n-2}\right)+\delta_{b}\left(v_{n-1}, v_{n-2}\right)\right) \\
& +\ldots+b^{n-m-1}\left(\delta_{b}\left(u_{m+2}, u_{m+1}\right)+\delta_{b}\left(v_{m+2}, v_{m+1}\right)\right) \\
& +b^{n-m}\left(\delta_{b}\left(u_{m+1}, u_{m}\right)+\delta_{b}\left(v_{m+1}, v_{m}\right)\right) \\
& \preceq b \delta_{n-1}+b^{2} \delta_{n-2}+\ldots+b^{n-m} \delta_{m} \\
& \preceq b\left(\left(2^{\frac{1}{2}} a\right)^{*}\right)^{n-1} \delta_{0}\left(2^{\frac{1}{2}} a\right)^{n-1}+b^{2}\left(\left(2^{\frac{1}{2}} a\right)^{*}\right)^{n-2} \delta_{0}\left(2^{\frac{1}{2}} a\right)^{n-2} \\
& +\ldots+b^{n-m}\left(\left(2^{\frac{1}{2}} a\right)^{*}\right)^{m} \delta_{0}\left(2^{\frac{1}{2}} a\right)^{m} \\
& \preceq \sum_{k=n-1}^{m} b^{n-k}\left(\left(2^{\frac{1}{2}} a\right)^{*}\right)^{k} \delta_{0}\left(2^{\frac{1}{2}} a\right)^{k} .
\end{aligned}
$$

\section{Therefore,}

$$
\begin{aligned}
&\left\|\delta_{b}\left(u_{n}, u_{m}\right)+\delta_{b}\left(v_{n}, v_{m}\right)\right\| \leq \sum_{k=n-1}^{m}\|b\|^{n-k}\|\sqrt{2} a\|^{2 k} \delta_{0} \\
& \leq \sum_{k=n-1}^{\infty}\|b\|^{n-k}\|\sqrt{2} a\|^{2 k} \delta_{0} \\
& \leq \frac{\|b\|\|\sqrt{2} a\|^{2(n-1)}}{1-\|b\|^{-1}\|\sqrt{2} a\|^{2}} \delta_{0} \\
&=\frac{\|b\|}{1-\|b\|^{-1}\|\sqrt{2} a\|^{2}}\|\sqrt{2} a\|^{2(n-1)} \delta_{0} . \\
& 730
\end{aligned}
$$


Since $\|a\|<\frac{1}{\sqrt{2}}$, we have

$$
\begin{aligned}
\left\|\delta_{b}\left(u_{n}, u_{m}\right)+\delta_{b}\left(v_{n}, v_{m}\right)\right\| & \leq \frac{\|b\|}{1-\|b\|^{-1}|| \sqrt{2} a \|^{2}}\|\sqrt{2} a\|^{2(n-1)} \delta_{0} \longrightarrow 0, \\
\delta_{b}\left(u_{n}, u_{m}\right) & \preceq \delta_{b}\left(u_{n}, u_{m}\right)+\delta_{b}\left(v_{n}, v_{m}\right),
\end{aligned}
$$

and

$$
\delta_{b}\left(v_{n}, v_{m}\right) \preceq \delta_{b}\left(v_{n}, v_{m}\right)+\delta_{b}\left(u_{n}, u_{m}\right),
$$

yields that $\left\{u_{n}\right\}$ and $\left\{v_{n}\right\}$ are Cauchy sequences in $\mathcal{X}$, so we can find $u$ and $v$ in $\mathcal{X}$ such that $\lim _{n \rightarrow \infty} u_{n}=u$ and $\lim _{n \rightarrow \infty} v_{n}=v$. Now, we prove that $\psi(u, v)=u$ and $\psi(v, u)=v$.

From the triangle inequality and (1), we obtain

$$
\begin{aligned}
\delta_{b}(\psi(u, v), u) & \preceq b\left[\delta_{b}\left(\psi(u, v), u_{n+1}\right)+\delta_{b}\left(u_{n+1}, u\right)\right] \\
& \preceq b\left[\delta_{b}\left(\psi(u, v), \psi\left(u_{n}, v_{n}\right)\right)+\delta_{b}\left(u_{n+1}, u\right)\right] \\
& \preceq b\left[a^{*} \delta_{b}\left(u, u_{n}\right) a+a^{*} \delta_{b}\left(v, v_{n}\right) a+\delta_{b}\left(u_{n+1}, u\right)\right],
\end{aligned}
$$

taking $n \rightarrow \infty$, we have that $\delta_{b}(\psi(u, v), u)=0_{\mathcal{A}}$, and consequently $\psi(u, v)=$ $u$. In the same way $\psi(v, u)=v$. Therefore, a coupled FP of $\psi$ is $(u, v)$.

Assume that another coupled FP of $\psi$ is $\left(u^{\prime}, v^{\prime}\right)$, so

$$
\begin{aligned}
& \delta_{b}\left(u, u^{\prime}\right)=\delta_{b}\left(\psi(u, v), \psi\left(u^{\prime}, v^{\prime}\right)\right) \preceq a^{*} \delta_{b}\left(u, u^{\prime}\right) a+a^{*} \delta_{b}\left(v, v^{\prime}\right) a, \\
& \delta_{b}\left(v, v^{\prime}\right)=\delta_{b}\left(\psi(v, u), \psi\left(v^{\prime}, u^{\prime}\right)\right) \preceq a^{*} \delta_{b}\left(v, v^{\prime}\right) a+a^{*} \delta_{b}\left(u, u^{\prime}\right) a,
\end{aligned}
$$

and hence

$$
\delta_{b}\left(u, u^{\prime}\right)+\delta_{b}\left(v, v^{\prime}\right) \preceq(\sqrt{2} a)^{*}\left(\delta_{b}\left(u, u^{\prime}\right)+\delta_{b}\left(v, v^{\prime}\right)\right)(\sqrt{2} a) .
$$

The above inequality with $\|\sqrt{2} a\|<1$ yields that

$$
\left\|\delta_{b}\left(u, u^{\prime}\right)+\delta_{b}\left(v, v^{\prime}\right)\right\| \leq\|\sqrt{2} a\|^{2}\left\|\delta_{b}\left(u, u^{\prime}\right)+\delta_{b}\left(v, v^{\prime}\right)\right\| .
$$

The above inequality holds only when $\left\|\delta_{b}\left(u, u^{\prime}\right)+\delta_{b}\left(v, v^{\prime}\right)\right\|=0$, which gives $\left(u^{\prime}, v^{\prime}\right)=(u, v)$. So the coupled FP is unique.

Now, we prove that $u=v$ to show that $\psi$ has a unique FP. Note that

$$
\delta_{b}(u, v)=\delta_{b}(\psi(u, v)+\psi(v, u)) \preceq a^{*} \delta_{b}(u, v) a+a^{*} \delta_{b}(v, u) a,
$$

and hence

$$
\left\|\delta_{b}(u, v)\right\| \leq\|a\|^{2}\left\|\delta_{b}(u, v)\right\|+\|a\|^{2}\left\|\delta_{b}(v, u)\right\|
$$




$$
\leq 2\|a\|^{2}\left\|\delta_{b}(u, v)\right\|
$$

In fact, from $\|a\|<\frac{1}{\sqrt{2}}$ we get $\left\|\delta_{b}(u, v)\right\|=0$, thus $u=v$.

Lemma 1. (Ma et al, 2014)

1) If $u \in \mathcal{A}_{+}$with $\|u\|<\frac{1}{2}$, then $1_{\mathcal{A}}-u$ is invertible.

2) If $u, v \in \mathcal{A}_{+}$with $u v=v u$, then $u v \succeq 0_{\mathcal{A}}$.

3) If $u, v \in \mathcal{A}_{h}$ and $t \in \mathcal{A}_{+}^{\prime}$, then $u \preceq v$ deduces $t u \preceq t v$, where $\mathcal{A}_{+}^{\prime}=$ $\mathcal{A}_{+} \cap \mathcal{A}^{\prime}$.

Theorem 2. Assume that $\left(X, \mathcal{A}, \delta_{b}\right)$ is a complete $C^{*}-A V-B M$ space and suppose that the function $\psi: \mathcal{X}^{2} \rightarrow \mathcal{X}$ satisfies

$$
\delta_{b}(\psi(u, v), \psi(t, s)) \preceq a \delta_{b}(\psi(u, v), u)+b \delta_{b}(\psi(t, s), t), \forall u, v, t, s \in \mathcal{X}
$$

in which $a, b, c, \in \mathcal{A}_{+}^{\prime}$ with $\|a\|+\|b\|<1,\|a c\|+\|b c\|<1,\|c\|>1$. Then $\psi$ has a unique coupled FP. Also, $\psi$ has a unique FP in $\mathcal{X}$.

Proof. As in Theorem 1, choose $\left\{u_{n}\right\}$ and $\left\{v_{n}\right\}$ in $\mathcal{X}$ and set $u_{n+1}=$ $\psi\left(u_{n}, v_{n}\right)$ and $v_{n+1}=\psi\left(v_{n}, u_{n}\right)$. Then from (4),

$$
\begin{aligned}
\delta_{b}\left(u_{n}, u_{n+1}\right) & =\delta_{b}\left(\psi\left(u_{n-1}, v_{n-1}\right), \psi\left(u_{n}, v_{n}\right)\right) \\
& \preceq a \delta_{b}\left(\psi\left(u_{n-1}, v_{n-1}\right), u_{n-1}\right)+b \delta_{b}\left(\psi\left(u_{n}, v_{n}\right), u_{n}\right) \\
& =a \delta_{b}\left(u_{n}, u_{n-1}\right)+b \delta_{b}\left(u_{n+1}, u_{n}\right),
\end{aligned}
$$

Thus,

$$
\left(1_{\mathcal{A}}-b\right) \delta_{b}\left(u_{n}, u_{n+1}\right) \preceq a \delta_{b}\left(u_{n}, u_{n-1}\right),
$$

Similarly,

$$
\left(1_{\mathcal{A}}-b\right) \delta_{b}\left(v_{n}, v_{n+1}\right) \preceq a \delta_{b}\left(v_{n}, v_{n-1}\right),
$$

Since $a, b \in \mathcal{A}_{+}^{\prime}$ with $\|a\|+\|b\|<1$, then $1_{\mathcal{A}}-b$ is invertible and $\left(1_{\mathcal{A}}-\right.$ $b)^{-1} a \in \mathcal{A}_{+}^{\prime}$. Therefore

$$
\begin{aligned}
\delta_{b}\left(u_{n}, u_{n+1}\right) & \preceq\left(1_{\mathcal{A}}-b\right)^{-1} a \delta_{b}\left(u_{n}, u_{n-1}\right), \\
\delta_{b}\left(v_{n}, v_{n+1}\right) & \preceq\left(1_{\mathcal{A}}-b\right)^{-1} a \delta_{b}\left(v_{n}, v_{n-1}\right),
\end{aligned}
$$

Then

$$
\left\|\delta_{b}\left(u_{n}, u_{n+1}\right)\right\| \leq\left\|\left(1_{\mathcal{A}}-b\right)^{-1} a\right\|\left\|\delta_{b}\left(u_{n}, u_{n-1}\right)\right\|,
$$




$$
\left\|\delta_{b}\left(v_{n}, v_{n+1}\right)\right\| \leq\left\|\left(1_{\mathcal{A}}-b\right)^{-1} a\right\|\left\|\delta_{b}\left(v_{n}, v_{n-1}\right)\right\|,
$$

It follows from the fact

$$
\left\|\left(1_{\mathcal{A}}-b\right)^{-1} a\right\| \leq\left\|\left(1_{\mathcal{A}}-b\right)^{-1} \mid\right\|\|a\| \leq \sum_{k=0}^{\infty}\|b\|^{k}\|a\|=\frac{\|a\|}{1-\|b\|}<1 .
$$

Hence $\left\{u_{n}\right\}$ and $\left\{v_{n}\right\}$ are Cauchy sequences in $\mathcal{X}$. By the completeness of $\mathcal{X}$, there are $u, v \in \mathcal{X}$ such that $\lim _{n \rightarrow \infty} u_{n}=u$ and $\lim _{n \rightarrow \infty} v_{n}=v$. As

$$
\begin{aligned}
\delta_{b}(\psi(u, v), u) & \preceq c\left[\delta_{b}\left(u_{n+1}, \psi(u, v)\right)+\delta_{b}\left(u_{n+1}, u\right)\right] \\
& =c \delta_{b}\left(\psi\left(u_{n}, v_{n}\right), \psi(u, v)\right)+c \delta_{b}\left(u_{n+1}, u\right) \\
& \preceq c a \delta_{b}\left(\psi\left(u_{n}, v_{n}\right), u_{n}\right)+c b \delta_{b}(\psi(u, v), u)+c \delta_{b}\left(u_{n+1}, u\right) \\
& \preceq c a \delta_{b}\left(u_{n+1}, u_{n}\right)+c b \delta_{b}(\psi(u, v), u)+c \delta_{b}\left(u_{n+1}, u\right),
\end{aligned}
$$

which deduces that

$$
\delta_{b}(\psi(u, v), u) \preceq\left(1_{\mathcal{A}}-c b\right)^{-1} c a \delta_{b}\left(u_{n+1}, u_{n}\right)+\left(1_{\mathcal{A}}-c b\right)^{-1} c \delta_{b}\left(u_{n+1}, u\right) .
$$

like above

$$
\begin{gathered}
\left\|\left(1_{\mathcal{A}}-c b\right)^{-1} c a\right\| \leq\left\|( 1 _ { \mathcal { A } } - c b ) ^ { - 1 } \left|\||| c a\| \leq \sum_{k=0}^{\infty}\|c b\|^{k}\|c a\|=\frac{\|c a\|}{1-\|c b\|}<1 .\right.\right. \\
\left\|\left(1_{\mathcal{A}}-c b\right)^{-1} c\right\| \leq\left\|\left(1_{\mathcal{A}}-c b\right)^{-1} \mid\right\|\|c\| \leq \sum_{k=0}^{\infty}\|c b\|^{k}\|c\|=\frac{\|c\|}{1-\|c b\|}<1 .
\end{gathered}
$$

Then $\delta_{b}(\psi(u, v), u)=0_{\mathcal{A}}$ or equivalently $\psi(u, v)=u$. In the same way, $\psi(v, u)=v$. Now if $\left(u^{\prime}, v^{\prime}\right)$ is another coupled FP of $\psi$, then from (4), we get

$$
\begin{aligned}
\delta_{b}\left(u, u^{\prime}\right) & =\delta_{b}\left(\psi(u, v), \psi\left(u^{\prime}, v^{\prime}\right)\right) \\
& \preceq a \delta_{b}\left(u^{\prime}, \psi\left(u^{\prime}, v^{\prime}\right)\right)+b \delta_{b}(u, \psi(u, v))=0_{\mathcal{A}},
\end{aligned}
$$

Hence $\delta_{b}\left(u^{\prime}, u\right)=0_{\mathcal{A}}$, and then $u^{\prime}=u$. In the same way, we can obtain that $v^{\prime}=v$. That is, $(u, v)$ is the unique coupled FP of $\psi$. Now, we prove the uniqueness of FPs of $\psi$. Using (4), we obtain

$$
\begin{aligned}
\delta_{b}(u, v) & =\delta_{b}(\psi(u, v), \psi(v, u)) \\
& \preceq a \delta_{b}(\psi(u, v), u)+b \delta_{b}(\psi(v, u), v) \\
& =a \delta_{b}(u, u)+b \delta_{b}(v, v)=0_{\mathcal{A}} .
\end{aligned}
$$

This yields that $u=v$. 
Theorem 3. Assume that $\left(\mathcal{X}, \mathcal{A}, \delta_{b}\right)$ is a complete $C^{*}-A V-B M$ space and let the function $F: \mathcal{X}^{2} \rightarrow \mathcal{X}$ hold

$$
\delta_{b}(\psi(u, v), \psi(t, s)) \preceq a \delta_{b}(\psi(u, v), t)+b \delta_{b}(\psi(t, s), u), \forall u, v, t, s \in \mathcal{X}
$$

where $a, b, c \in \mathcal{A}_{+}^{\prime}$ with $\|a\|+\|b\|<1,\|a c\|+\|b c\|<1,\|c\|>1$. then $\psi$ has a unique coupled FP. Also, $\psi$ has a unique FP in $\mathcal{X}$.

Proof. Similar to Theorem 2, choose two sequences $\left\{u_{n}\right\}$ and $\left\{v_{n}\right\}$ in $\mathcal{X}$ and set $u_{n+1}=\psi\left(u_{n}, v_{n}\right)$ and $v_{n+1}=\psi\left(v_{n}, u_{n}\right)$. Then from (5) we obtain

$$
\begin{aligned}
\delta_{b}\left(u_{n}, u_{n+1}\right) & =\delta_{b}\left(\psi\left(u_{n-1} v_{n-1}\right), \psi\left(u_{n}, v_{n}\right)\right) \\
& \preceq a \delta_{b}\left(\psi\left(u_{n-1}, v_{n-1}\right), u_{n}\right)+b \delta_{b}\left(\psi\left(u_{n}, v_{n}\right), u_{n-1}\right) \\
& =a \delta_{b}\left(u_{n}, u_{n}\right)+b \delta_{b}\left(u_{n+1}, u_{n-1}\right) \\
& =b \delta_{b}\left(u_{n+1}, u_{n-1}\right) \\
& \preceq c b\left(\delta_{b}\left(u_{n+1}, u_{n}\right)+\delta_{b}\left(u_{n}, u_{n-1}\right)\right) \\
& =c b \delta_{b}\left(u_{n+1}, u_{n}\right)+c b \delta_{b}\left(u_{n}, u_{n-1}\right),
\end{aligned}
$$

Thus,

$$
\left(1_{\mathcal{A}}-c b\right) \delta_{b}\left(u_{n}, u_{n+1}\right) \preceq c b \delta_{b}\left(u_{n}, u_{n-1}\right) .
$$

By the symmetry in (5),

$$
\begin{aligned}
\delta_{b}\left(u_{n+1}, u_{n}\right) & =\delta_{b}\left(\psi\left(u_{n} v_{n}\right), \psi\left(u_{n-1}, v_{n-1}\right)\right) \\
& \preceq a \delta_{b}\left(\psi\left(u_{n}, v_{n}\right), u_{n-1}\right)+b \delta_{b}\left(\psi\left(u_{n-1}, v_{n-1}\right), u_{n}\right) \\
& =a \delta_{b}\left(u_{n+1}, u_{n-1}\right)+b \delta_{b}\left(u_{n}, u_{n}\right) \\
& =a \delta_{b}\left(u_{n+1}, u_{n-1}\right) \\
& \preceq c a\left(\delta_{b}\left(u_{n+1}, u_{n}\right)+\delta_{b}\left(u_{n}, u_{n-1}\right)\right) \\
& =c a \delta_{b}\left(u_{n+1}, u_{n}\right)+c a \delta_{b}\left(u_{n}, u_{n-1}\right),
\end{aligned}
$$

that is,

$$
\left(1_{\mathcal{A}}-c a\right) \delta_{b}\left(u_{n}, u_{n+1}\right) \preceq c a \delta_{b}\left(u_{n}, u_{n-1}\right) .
$$

Now, from (6) and (7) we obtain

$$
\left(1_{\mathcal{A}}-\frac{c a+c b}{2}\right) \delta_{b}\left(u_{n}, u_{n+1}\right) \preceq \frac{c a+c b}{2} \delta_{b}\left(u_{n}, u_{n-1}\right) .
$$


Since $a, b, c \in \mathcal{A}_{+}^{\prime}$ with $\|c a+c b\| \leq\|c a\|+\|c b\|<1$, then $\left(1_{\mathcal{A}}-\frac{c a+c b}{2}\right)^{-1} \in$ $\mathcal{A}_{+}^{\prime}$, which together with [3, Lemma 1] yields that

$$
\delta_{b}\left(u_{n}, u_{n+1}\right) \preceq\left(1_{\mathcal{A}}-\frac{c a+c b}{2}\right)^{-1} \frac{c a+c b}{2} \delta_{b}\left(u_{n}, u_{n-1}\right) .
$$

Let $t=\left(1_{\mathcal{A}}-\frac{c a+c b}{2}\right)^{-1} \frac{c a+c b}{2}$, then $\|t\|=\left\|\left(1_{\mathcal{A}}-\frac{c a+c b}{2}\right)^{-1} \frac{c a+c b}{2}\right\|<1$.

By using the same argument of Theorem 2, we obtain $\left\{u_{n}\right\}$ which is a Cauchy sequence in $X$. Also, one can show that $\left\{v_{n}\right\}$ is a Cauchy sequence in $\mathcal{X}$. Therefore by the completeness of $\mathcal{X}$, there are $u, v \in \mathcal{X}$ such that $\lim _{n \rightarrow \infty} u_{n}=u$ and $\lim _{n \rightarrow \infty} v_{n}=v$. Now, we obtain that $\psi(u, v)=u$ and $\psi(v, u)=v$. To do this, we have

$$
\begin{aligned}
\delta_{b}(\psi(u, v), u) & \preceq c\left[\delta_{b}\left(u_{n+1}, \psi(u, v)\right)+\delta_{b}\left(u_{n+1}, u\right)\right] \\
& =c \delta_{b}\left(\psi\left(u_{n}, v_{n}\right), \psi(u, v)\right)+c \delta_{b}\left(u_{n+1}, u\right) \\
& \preceq c a \delta_{b}\left(\psi\left(u_{n}, v_{n}\right), u\right)+c b \delta_{b}\left(\psi(u, v), u_{n}\right)+c \delta_{b}\left(u_{n+1}, u\right) \\
& \preceq c a \delta_{b}\left(u_{n+1}, u\right)+c b \delta_{b}\left(\psi(u, v), u_{n}\right)+c \delta_{b}\left(u_{n+1}, u\right),
\end{aligned}
$$

and then

$\left\|\delta_{b}(\psi(u, v), u)\right\| \leq\left\|c a\left|\left\||| \delta_{b}\left(u_{n+1}, u\right)\right\|+\left\|c b|||| \delta_{b}\left(\psi(u, v), u_{n}\right)\right\|+\| c\right|||\left|\delta_{b}\left(u_{n+1}, u\right)\right| \mid\right.$

by the continuity of the metric and the norm, we get

$$
\left\|\delta_{b}(\psi(u, v), u)\right\| \leq\left\|c b \left|\left\||| \delta_{b}(\psi(u, v), u)\right\| .\right.\right.
$$

Since $\|c b\|<1$, it implies that $\left\|\delta_{b}(\psi(u, v), u)\right\|=0$, and then $\psi(u, v)=u$. In the same way, $\psi(v, u)=v$, which implies that $(u, v)$ is a coupled FP of $\psi$. By the same reasoning in Theorem 2, we obtain $u=v$, which means that $\psi$ has a unique FP in $\mathcal{X}$.

\section{Existence and uniqueness}

Consider the next equation:

$$
x(m)=\int_{\mathcal{E}}\left(T_{1}(m, n)+T_{2}(m, n)\right)(\alpha(n, x(n))+\beta(n, x(n)) d n+J(m), m \in \mathcal{E}
$$

for the Lebesgue measurable set of $\mathcal{E}$ in which $m(\mathcal{E})<\infty$. 
In what follows, we always let $\mathcal{X}=L^{\infty}(\mathcal{E})$ denote a class of essentially bounded measurable functions on $\mathcal{E}$, where $\mathcal{E}$ is a Lebesgue measurable set such that $m(\mathcal{E})<\infty$.

Now, we consider the functions $T_{1}, T_{2}, \alpha, \beta$ fulfill the following assumptions:

(i) $T_{1}$ from $\mathcal{E} \times \mathcal{E}$ to $\mathbb{R}^{\geq 0}, T_{2}$ from $\mathcal{E} \times \mathcal{E}$ to $\mathbb{R} \leq 0$. Also, two integrable functions $\alpha$ and $\beta$ are from $\mathcal{E} \times \mathbb{R}$ to $\mathbb{R}$, and $J \in L^{\infty}(\mathcal{E})$;

(ii) there exists $\ell \in\left(0, \frac{1}{2}\right)$ such that

$$
0 \leq \alpha(m, x)-\alpha(m, y) \leq \ell(x-y)
$$

and

$$
-\ell(x-y) \leq \beta(m, x)-\beta(m, y) \leq 0
$$

for $m \in \mathcal{E}$ and $x, y \in \mathbb{R}$;

(iii) $\sup _{m \in \mathcal{E}} \int_{\mathcal{E}}\left(T_{1}(m, n)-T_{2}(m, n)\right) d n \leq 1$.

Theorem 4. Suppose that assumptions (i)-(iii) hold. Then the integral equation (8) has a unique solution in $L^{\infty}(\mathcal{E})$.

Proof. Let $\mathcal{X}=L^{\infty}(\mathcal{E})$ and $B\left(L^{2}(\mathcal{E})\right)$ be the set of bounded linear operators on a Hilbert space $L^{2}(\mathcal{E})$. We endow $\mathcal{X}$ with the b-metric $\delta_{b}: \mathcal{X} \times \mathcal{X} \rightarrow$ $B\left(L^{2}(\mathcal{E})\right)$ defined by

$$
\delta_{b}(\alpha, \beta)=M_{|\alpha-\beta|^{p}}
$$

where $M_{|\alpha-\beta|^{p}}$ is the multiplication operator on $L^{2}(\mathcal{E})$. Hence $\left(\mathcal{X}, B\left(L^{2}(\mathcal{E})\right), \delta_{b}\right)$ is a complete $\mathrm{C}^{*}$-AV-BM space. Define the self-mapping $\Psi: \mathcal{X} \times \mathcal{X} \rightarrow \mathcal{X}$ by

$$
\begin{aligned}
\Psi(x, y)(m) & =\int_{\mathcal{E}} T_{1}(m, n)(\alpha(n, x(n))+\beta(n, y(n))) d n \\
& +T_{2}(m, n)(\alpha(n, y(n))+\beta(n, x(n))) d n+J(m),
\end{aligned}
$$

for all $m \in \mathcal{E}$. Now, we have

$$
\delta_{b}(\Psi(x, y), \Psi(u, v))=M_{|\Psi(x, y)-\Psi(u, v)|^{p} .}
$$

We obtain,

$$
|(\Psi(x, y)-\Psi(u, v))(m)|^{p}=\mid \int_{\mathcal{E}} T_{1}(m, n)(\alpha(n, x(n))+\beta(n, y(n))) d n
$$




$$
\begin{aligned}
+ & \int_{\mathcal{E}} T_{2}(m, n)(\alpha(n, y(n))+\beta(n, x(n))) d n-\int_{\mathcal{E}} T_{1}(m, n)(\alpha(n, u(n)) \\
+ & \beta(n, v(n))) d n-\left.\int_{\mathcal{E}} T_{2}(m, n)(\alpha(n, v(n))+\beta(n, u(n))) d n\right|^{p} \\
= & \left(\left|\int_{\mathcal{E}} T_{1}(m, n)(\alpha(n, x(n))-\alpha(n, u(n))+\beta(n, y(n))-\beta(n, v(n))) d n\right|\right. \\
+ & \left.\left|\int_{\mathcal{E}} T_{2}(m, n)(\alpha(n, y(n))-\alpha(n, v(n))+\beta(n, x(n))-\beta(n, u(n))) d n\right|\right)^{p} \\
\leq & \left(\int_{\mathcal{E}} T_{1}(m, n)|\alpha(n, x(n))-\alpha(n, u(n))+\beta(n, y(n))-\beta(n, v(n))| d n\right. \\
- & \left.\int_{\mathcal{E}} T_{2}(m, n)|\alpha(n, y(n))-\alpha(n, v(n))+\beta(n, x(n))-\beta(n, u(n))| d n\right)^{p} \\
\leq & \left(\sup _{n \in \mathcal{E}}[\ell|x(n)-u(n)|+\ell|y(n)-v(n)|] \times \int_{\mathcal{E}}\left(T_{1}(m, n)-T_{2}(m, n)\right) d n\right)^{p} \\
\leq & \left(\left[\ell\|x-u\|_{\infty}+\ell\|y-v\|_{\infty}\right] \sup _{m \in \mathcal{E}} \int_{\mathcal{E}}\left(T_{1}(m, n)-T_{2}(m, n)\right) d n\right)^{p} \\
\leq & \left(\ell\|x-u\|_{\infty}+\ell\|y-v\|_{\infty}\right)^{p} \\
\leq & \ell\left(\|x-u\|_{\infty}+\|y-v\|_{\infty}\right)^{p} .
\end{aligned}
$$

Therefore,

$$
\begin{aligned}
\left\|\delta_{b}(\Psi(x, y), \Psi(u, v))\right\| & =\left\|M_{|\Psi(x, y)-\Psi(u, v)|^{p}}\right\| \\
& =\sup _{\|\varphi\|=1}\left(M_{\left.|\Psi(x, y)-\Psi(u, v)|^{p} \varphi, \varphi\right)}\right. \\
& =\sup _{\|\varphi\|=1} \int_{\mathcal{E}}|(\Psi(x, y)-\Psi(u, v))(m)|^{p} \varphi(m) \varphi(m) d m \\
& \leq \sup _{\|\varphi\|=1} \int_{\mathcal{E}}|\varphi(m)|^{2} d t\left(\ell\|x-u\|_{\infty}+\ell\|y-v\|_{\infty}\right)^{p} \\
& \leq\left(\ell\|x-u\|_{\infty}+\ell\|y-v\|_{\infty}\right)^{p} \\
& \leq \ell\left(\|x-u\|_{\infty}+\|y-v\|_{\infty}\right)^{p} .
\end{aligned}
$$

Set $a=\sqrt{\ell} 1_{B\left(L^{2}(\mathcal{E})\right)}$, then $a \in B\left(L^{2}(\mathcal{E})\right)$ and $\|a\|=|\sqrt{\ell}|<\frac{1}{\sqrt{2}}$. Hence by applying Theorem 1 , we get the desired result.

\section{Funding}

No funding was received. 


\section{Competing interests}

The authors declare that they have no competing interests.

\section{Authors' contributions}

All authors contributed to the study, participated in its design and coordination, drafted the manuscript, participated in the sequence alignment, and read and approved the final manuscript.

\section{References}

Ali Abou Bakr, S. M. 2019. Common fixed point of generalized cyclic Banach algebra contractions and Banach algebra Kannan types of mappings on cone quasi metric spaces. Journal of Nonlinear Sciences and Applications (JNSA), 12(10), pp.644-655. Available at: http://dx.doi.org/10.22436/jnsa.012.10.03.

Bai, C. 2016. Coupled fixed point theorems in $C^{*}$-algebra-valued b-metric spaces with application. Fixed Point Theory and Applications, art.number:70. Available at: https://doi.org/10.1186/s13663-016-0560-1.

Bonsal, F.F. 1962. Lectures On Some Fixed Point Theorems Of Functional Analysis. Bombay, India: Tata Institute Of Fundamental Research [online]. Available at: http://www.math.tifr.res.in/ publ/In/tifr26.pdf. [Accessed: 25 September 2020].

Cao, T. and Xin, Q. 2016. Common coupled fixed point theorems in $C^{*}$ algebra-valued metric spaces. arXiv:1601.07168v1[math.OA], 26 January [online]. Available at: https://arxiv.org/abs/1601.07168 [Accessed: 25 September 2020].

Huang, H., Došenović, T. and Radenović, S. 2018. Some fixed point results in b-metric spaces approach to the existence of a solution to nonlinear integral equations. Journal of Fixed Point Theory and Applications, 20(art.number:105). Available at: https://doi.org/10.1007/s11784-018-0577-7.

Hussain, A., Kanwal, T., Mitrović, Z. D. and Radenović, S., 2018. Optimal Solutions and Applications to Nonlinear Matrix and Integral Equations via Simulation Function. Filomat, 32(17), pp.6087-6106. Available at: https://doi.org/10.2298/FI L1817087H.

Hussain, N. and Mitrović, Z. D. 2017. On multi-valued weak quasi-contractions in b-metric spaces. Journal of Nonlinear Sciences and Applications (JNSA), 10(7), pp.3815-3823. Available at: http://dx.doi.org/10.22436/jnsa.010.07.35.

Kadelburg, Z., Nastasi, A., Radenović, S. and Vetro, P. 2016. Fixed points of contractions and cyclic contractions on $C *$-algebra-valued b-metric spaces. $A d$ vances in Operator Theory, 1(1), pp.92-103. Available at: https://doi.org/10.22034 laot.1610.1030. 
Kadelburg, Z. and Radenović, S., 2016. Fixed point results in $C *$-algebravalued metric spaces are direct consequences of their standard metric counterparts. Fixed Point Theory and Applications, art.number:53(2016). Available at: https://doi.org/10.1186/s13663-016-0544-1.

Kongban, C. and Kumam, P. 2018. Quadruple random common fixed point results of generalized Lipschitz mappings in cone b-metric spaces over Banach algebras. Journal of Nonlinear Sciences and Applications (JNSA), 11(1), pp.131149. Available at: http://dx.doi.org/10.22436/jnsa.011.01.10.

Ma, Z. and Jiang, L. 2015. $C^{*}$-Algebra-valued b-metric spaces and related fixed point theorems. Fixed Point Theory and Applications , art.number:222(2015). Available at: https://doi.org/10.1186/s13663-015-0471-6.

Ma, Z.H., Jiang, N. and Sun, H.K. 2014. $C^{*}$-Algebra-valued metric spaces and related fixed point theorems. Fixed Point Theory and Applications, art.number:206(2014). Available at: https://doi.org/10.1186/1687-1812-2014-206.

Mitrović, Z.D., Aydi, H., Md Noorani, M.S. and Qawaqneh, H. 2019. The weight inequalities on Reich type theorem in b-metric spaces. Journal of Mathematics and Computer Science (JMCS), 19(1), pp.51-57. Available at: http://dx.doi.org/10.22 436/jmcs.019.01.07.

Radenović, S., Vetro, P., Nastasi, A. and Quan, L.T. 2017. Coupled fixed point theorems in $C *$-algebra-valued b-metric spaces. Scientific Publications of the State University of Novi Pazar Series A: Applied Mathematics, Informatics and mechanics, 9(1), pp.81-90. Available at: https://doi.org/10.5937/SPSUNP1701081R.

Radenović, S., Zoto, K., Dedović, N., Šešum Čavić, V. and Ansari, A.H. 2019. Bhaskar-Guo-Lakshmikantam-Ćirić type results via new functions with applications to integral equations. Applied Mathematics and Computation, 357(C), pp.75-87. Available at: https://doi.org/10.1016/j.amc.2019.03.057.

Todorčević, V. 2019. Harmonic Quasiconformal Mappings and Hyperbolic Type Metrics. Springer Nature Switzerland AG. ISBN: 978-3-030-22591-9.

Vujaković, J., Kishore, G.N.V., Rao, K.P.R., Radenović, S. and Sadik, S.K. 2019. Existence and Unique Coupled Solution in $S_{b}$-Metric Spaces by Rational Contraction with Application. Mathematics, 7(4), p.313. Available at: https://doi. org/10.3390/math7040313.

Zoto, K., Rhoades, B.E. and Radenović, S. 2019. Common fixed point theorems for a class of $(s, q)$-contractive mappings in b-metric-like spaces and applications to integral equations. Mathematica Slovaca, 69(1), pp.233-247. Available at: https://doi.org/10.1515/ms-2017-0217.

$\mathrm{Wu}, \mathrm{X}$. and Zhao, L. 2019. Fixed point theorems for generalized $\alpha-\psi$ type contractive mappings in b-metric spaces and applications,. Journal of Mathematics and Computer Science (JMCS), 18(1), pp.49-62. Available at: http://dx.doi.org/10. 22436/jmcs.018.01.06. 


\section{СУЩЕСТВОВАНИЕ И ЕДИНСТВЕННОСТЬ РЕШЕНИЙ НЕКОТОРЫХ КЛАССОВ ИНТЕГРАЛЬНЫХ УРАВНЕНИЙ С*- АЛГЕБРЫ В $b$-МЕТРИЧЕСКИХ ПРОСТРАНСТВАХ}

Есад Якупович ${ }^{a}$, Хашем П. Масиха ${ }^{\sigma}$, Зоран Д. Митрович ${ }^{\text {, }}$ корессподент, Сеиде С. Разави ${ }^{б}$, Реза Саадатиг

а Академия наук и художеств Республики Сербской, г. Баня-Лука, Республика Сербская, Босния и Герцеговина

${ }^{б}$ Технологический университет «К. Н. Тооси», математический фракультет, г. Тегеран, Исламская Республика Иран

вУниверситет в г. Баня-Лука, электротехнический факультет, г. Баня-Лука, Республика Сербская, Босния и Герцеговина

'Иранский научно-технологический университет, математический колледж, Нармак, г. Тегеран, Исламская Республика Иран

РУБРИКА ГРНТИ: 27.00.00 МАТЕМАТИКА:

27.25.17 Метрическая теория функций,

27.33.00 Интегральные уравнения,

27.39.29 Приближенные методы

функционального анализа

ВИД СТАТЬИ: оригинальная научная статья

Резюме:

Введение/цель: Цель данной статьи заключается в выявлении сопряженных неподвижных точек $C$ *-алгебры в $b$ метрических пространствах. На основании полученных результатов обсуждается существование решений интегральных уравнений.

Методы: C помощью методов сопряжения неподвижных точек представлены необходимые условия для существования решений некоторых классов интегральных уравнений.

Результаты: Получены новые результаты о сопряженных неподвижных точках $C$ *-алгебры в $b$-метрическом пространстве.

Выводы: Полученные результаты вносят вклад в теорию неподвижных точек и открывают новые возможности применения в теории диффференциальных и интегральных уравнений.

Ключевые слова: сопряженная неподвижная точка, C *алгебра, интегральное уравнение. 


\section{ЕГЗИСТЕНЦИЈА И ЈЕДИНСТВЕНОСТ РЈЕШЕЊА НЕКИХ КЛАСА ИНТЕГРАЛНИХ ЈЕДНАЧИНА У $b$-МЕТРИЧКИМ ПРОСТОРИМА НАД $C^{*}$-АЛГЕБРАМА}

Есад Јакуповић ${ }^{a}$, Hashem P. Masiha ${ }^{\sigma}$, Зоран Д. Митровић ${ }^{\mathrm{B}}$, аутор за преписку, Seyede S. Razavi ${ }^{6}$, Reza Saadati

\footnotetext{
а Академија наука и умјетности Републике Српске, Бања Лука, Република Српска, Босна и Херцеговина

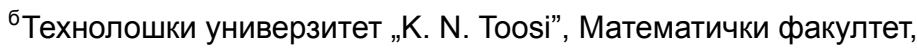
Техеран, Исламска Република Иран

вУниверзитет у Бањој Луци, Електротехнички факултет, Бања Лука, Република Српска, Босна и Херцеговина

'Ирански научно-технолошки универзитет, Колеџ математике, Нармак, Техеран, Исламска Република Иран

ОБЛАСТ:

математика

ВРСТА ЧЛАНКА: оригинални научни рад
}

Сажетак:

Увод/циљ: Циљ овог рада јесте да се добију одређени резултати о придруженим непокретним тачкама у $C$ *-алгебра b-метричким просторима. Користећи ове резултате дати су довољни услови за егзистенцију рјешења неких класа интегралних једначина.

Meтоде: Коришћењем методе придружених фриксних тачака дати су довољни услови за егзистенцију рјешења неких класа интегралних једначина.

Резултати: Добијени су нови резултати о придруженим фоиксним тачкама у b-метричком простору над $C^{*}$ алгебром.

Закључак: Добијени резултати представљају допринос теорији фиксних тачака и отварају нове могућности за примене у теорији дифреренцијалних и интегралних једначина.

Кључне речи: придружена фриксна тачка, C *-алгебра, интегрална једначина.

Paper received on / Дата получения работы / Датум пријема чланка: 30.09.2020. Manuscript corrections submitted on / Дата получения исправленной версии работы / Датум достављања исправки рукописа: 15.10.2020.

Paper accepted for publishing on / Дата окончательного согласования работы / Датум коначног прихватања чланка за објављивање: 16.10.2020. 
(c) 2019 The Authors. Published by Vojnotehnički glasnik / Military Technical Courier (http://vtg.mod.gov.rs, http://втг.мо.упр.срб). This article is an open access article distributed under the terms and conditions of the Creative Commons Attribution license (http://creativecommons.org/licenses/by/3.0/rs/).

(c) 2019 Авторы. Опубликовано в "Военно-технический вестник / Vojnotehnički glasnik / Military Technical Courier" (http://vtg.mod.gov.rs, http://втг.мо.упр.срб). Данная статья в открытом доступе и распространяется в соответствии с лицензией "Creative Commons" (http://creativecommons.org/licenses/by/3.0/rs/).

() 2019 Аутори. Објавио Војнотехнички гласник / Vojnotehnički glasnik / Military Technical Courier (http://vtg.mod.gov.rs, http://втг.мо.упр.срб). Ово је чланак отвореног приступа и дистрибуира се у складу са Creative Commons лиценцом (http://creativecommons.org/licenses/by/3.0/rs/). 